\title{
EnterococcalInfections And Its Antimicrobial Resistance With Special Reference To VRE And HLAR In A Tertiary Care Hospital In Eastern India
}

\author{
Ghosh(Ray)Reena ${ }^{1}$, Chatterjee Sumanta ${ }^{2}$, Bhattacharya Kumkum ${ }^{3}$, \\ Bhattacharya Sujata ${ }^{4}$. \\ ${ }^{I}$ (Deptt.of Microbiology; R G Kar Medical College \& Hospital, Kolkata, India.) \\ ${ }^{2}$ (Deptt.of Microbiology; $R$ G Kar Medical College \& Hospital, Kolkata, India.) \\ ${ }^{3}$ (Deptt.of Microbiology; $R$ G Kar Medical College \& Hospital, Kolkata, India.) \\ ${ }^{4}$ (Deptt.of Microbiology; $R$ G Kar Medical College \& Hospital, Kolkata, India.)
}

\begin{abstract}
Enterococci have traditionally been regarded as low grade pathogen, have emerged as an increasingly important cause of nosocomial infections in the last decade.Despite increasing reports of VRE in different countries ,there is scanty data on this issue from india especially in the Eastern zone.

A total of 157 enterococci were isolated from various clinical specimens (urine,pus\& wound swabs,blood and throat swabs body fluids), received in the Microbiology laboratory of a tertiary care hospital in eastern India during the period between July2010 to June2011.

Out of 157 enterococcal isolates52 showed vancomycin resistance by Kirby Bauer disc diffusion method. Of the 52 isolates 11 showed vancomycin resistance with an MIC > $4 \mu \mathrm{g} / \mathrm{ml}$ and 2 showed teicoplanin resistance with an MIC $>8 \mu \mathrm{g} / \mathrm{ml}$ done by agar dilution method following CLSI guidelines. Thelevel of resistance were further evaluated by determining MIC using " $E$ " Test strip of vancomycin and teicoplanin. Out of 11 VancomycinResistance Enterococci (VRE), 9 were characterized as VanB and 2 were found to be VanA phenotype.Out of 10 uncommon enterococcal isolates(07E.casseliflavus\& 03 E.gallinarum) 09 showed increased MIC to vancomycin $(02-04 \mu \mathrm{g} / \mathrm{ml}) .41 .4 \%$ isolates showed high level aminoglycoside resistance.
\end{abstract}

Key Words: Enterococci, Disc diffusion, MIC, antibiotic resistance, phenotype, VRE(vancomycin resistant enterococci), HLAR(High level aminoglycoside resistance).

\section{Introduction}

Enterococci are facultative anaerobes that are part of normal intestinal flora in humans[1,2].Sites less often colonized by enterococci include the oral cavity,genitourinary tract and skin especially in the perianal area.The main sites of colonization in the hospitalized patients are soft tissue wounds, ulcers and gastrointestinal tract(GIT)[3]Enterococci were traditionally regarded as low grade pathogens but have emerged as an increasingly important cause of nosocomial infections in the 1990s. These infections are recognized by3ts-tough ,tenacious and oftentimes troublesome[4].Though they are not considered to be highly virulent, their intrinsic resistance and ability to acquire resistance to several broad-spectrum antibiotics allows them to cause super infections in patients already receiving antimicrobial therapy[1,5,6].The increasing occurrence of E.faecium is of particular concern due to high resistance to antibiotics especially in nosocomial settings[7,8].Prior to 1990s also enterococci have been recognized as an important cause of bacterial endocarditis for almost a century[3,9].However, during the past decade, there has been a worldwide trend in increasing occurrence of enterococci(in the hospitals), a shift in the spectrum of enterdcoccal infections , and emergence of antimicrobial resistance among such isolates [9]. Enterococci were reported as the second leading cause of nosocomial infections[10].The most frequent infections caused by enterococci are urinary tract infections(UTIs)[3,9].The second most frequent enterococcal infections generally have been intra-abdominal and intra-pelvic abscesses or post surgery wound infections[3,10].The third most frequent infection caused by these organisms is blood stream infections (BSIs)[11].Other infections caused with lower frequency are central nervous system(CNS) and neonatal infections[11].

Enterococci have an acquired resistance to several antibiotics either by mutation or by receipt of foreign genetic material through the transfer of plasmids and transposons[2,18]. The acquisition of high level aminoglycoside resistance and vancomycin resistance has limited the therapeutic options available for clinicians [18]. The transfer potential ofvancomycin resistant genes from enterococci to Staphylococusaureus again increases the importance of finding ways to limit the spread of vancomycin resistant enterococi (VRE).

The present study was undertaken with the objective to study isolation, speciation and characterization of enterococci from clinical specimens, to determine the antimicrobial susceptibilitypattern of the isolates and to determine the phenotype of VRE and HLAR among the isolates. 


\section{Material \& Methods}

The present study was conducted in the department of Microbiology , RG.KarMedical college Kolkata which is a tertiary care hospital in Eastern India. A total of 157 Enterococcal strains were isolated from clinical samples namely - urine(73), wound swab\& pus(64), blood(10), throat swab(6), others(4) during the period between June 2010 to July2011. Strains isolated were identified according to standard laboratory procedures as per the scheme of Facklam\&CollinS(12) and also by using KB005 Hi Strep Identification kit (Hi Media Laboratories, Mumbai).

Antimicrobial susceptibility testing was done by Kirby- Baur disc diffusion method as perrecommendations of CLSI(13).Various antibiotic tested were:

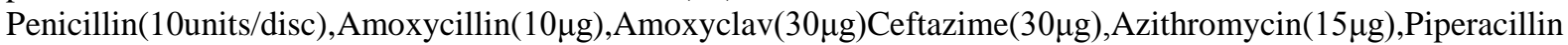

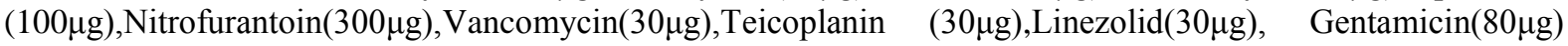
$\&$ Teigecycline $(30 \mu \mathrm{g})$.

The Vancomycin and Teicoplanin resistant strains identified by Kirby- Bauer disc diffusion method were further confirmed by Agar dilution method by supplementing Mueller-Hinton agar withVancomycin $6 \mu \mathrm{g} / \mathrm{ml}$ and Teicoplanin $8 \mu \mathrm{g} / \mathrm{ml}$ respectively asper CLSI recommendations(14).

MIC detection was done by using "E" test strip (Manufacturer-Bio Merieux,AB Biodisk) for Vancomycin among 11 VRE isolates and twoTeicoplanin resistant isolates (detected by agar dilution screening method) for MIC values of $0.125-256 \mu \mathrm{g} / \mathrm{ml}$.

Additionally MIC determination by "E" Test strip were performed for Vancomycin\&Teicoplaninamong ten(10) uncommon Enterococcal isolates identified (7 strains of E.casseliflavus\& 3 strains of E. gallinarum) which are known to demonstrate intrinsic, low level resistance to Vancomycinbut are susceptible to Teicoplanin.

High level aminoglycoside resistance (HLAR) was detected by agar dilution method for Gentamicin and Streptomycin by supplementing Mueller Hinton agar with $500 \mu \mathrm{g} / \mathrm{ml}$ and $2000 \mu \mathrm{g} / \mathrm{ml}$ antibiotics respectively. The source of media and antibiotic discs was Hi-Media Ltd.(Mumbai) India. The Standard strain E.faecalisATCC 29212 was used as negative control and ATCC 51299 was used as positive control.

\section{Results}

A total of 157 strains of enterococci were obtained from various clinical samples.Seventy three (46.49\%) strains were isolated from urine samples,64(40.76\%) strains were from pus\& wound swab samples, $10(6.36 \%)$ strains were from blood samples,6(3.82\%) strains were from throat swab samples and 4(2.54\%) strains were from other samples(BAL fluid\&other body fluids)[Table-1].

Various species of enterococci isolated were - E.fecalis 94(59.87\%), E. faecium 25(15.92\%), E. dispar15(9.55\%), E. durans8(5.0\%), E. casseliflavus 7(4.45\%), Emundtii5(3.18\% ),E.gallinarum3(1.91\%)[Table-1].

Antibiotic susceptibility testing showed increased resistance to various antibiotics tested[Table-2].By Kirby- Bauer disc diffusion method 52Enterococcal strains were found to be resistant toVancomycin and only two strains were found to be Teicoplanin resistant. All the strains were sensitive to Linezolid and Teigecycline. Interestingly all the strains (total-157 Enterococci) showed low level resistance to Gentamicin $(80 \mu \mathrm{g})$.

High level aminoglycoside resistance Streptomycin and Gentamicin were detected among 157 Enterococcal isolates. A total of 65 (41.4\%) isolates showed high level resistance to Gentamicin and / or Streptomycin [Table3].

Out of 52 VRE strains identifiedby disc diffusion method, 11 enterococcal strains were found to be Vancomycin resistant with MIC $>06 \mu \mathrm{g} / \mathrm{ml}$ by Vancomycin agar dilution method.

MIC determination by "E" Tests( AB Biodisk, bio Merieux) were done for eleven(11)strains identified by Vancomycinagardiluion method and Two(02) E.faecalis strains which showed Teicoplanin resistance both identified by disc diffusion and agar dilution method as shown in table[Table-4]. Both strains of E.faecalis showed MIC values of Vancomycin as $256 \mu \mathrm{g} / \mathrm{ml}$ (Figure 1). Teicoplanin as $16 \mu \mathrm{g} / \mathrm{ml}$.

Again, out Of Ten(10) uncommon enterococcal strains, nine(09)( E.casseliflavus 06\&E.gallinarum(03) showed

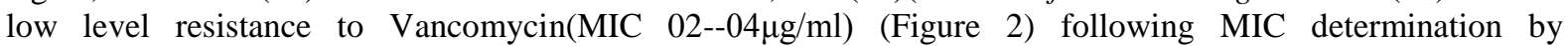
Vancomycin "E" Test strip and their MIC to Teicoplanin were also determined[Table-4 ].

Phenotypic characterization of Glycopeptideresistantance inenterococcal strains (VRE) based on the MIC values of Vancomycin and Teicoplanin obtained were depicted in table below[Table-4 ] 
Pie Diagram showing percentage distribution of enterococcal species

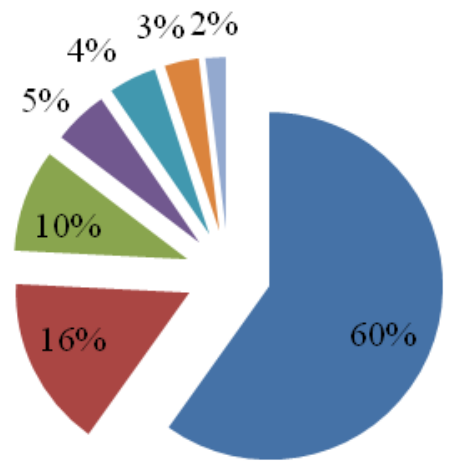

E.faecalis

E.faecium

E. dispar

E. durans

E. casseli

E.mundtii

E.galinarum

Table -1 :Source and speciation of isolates

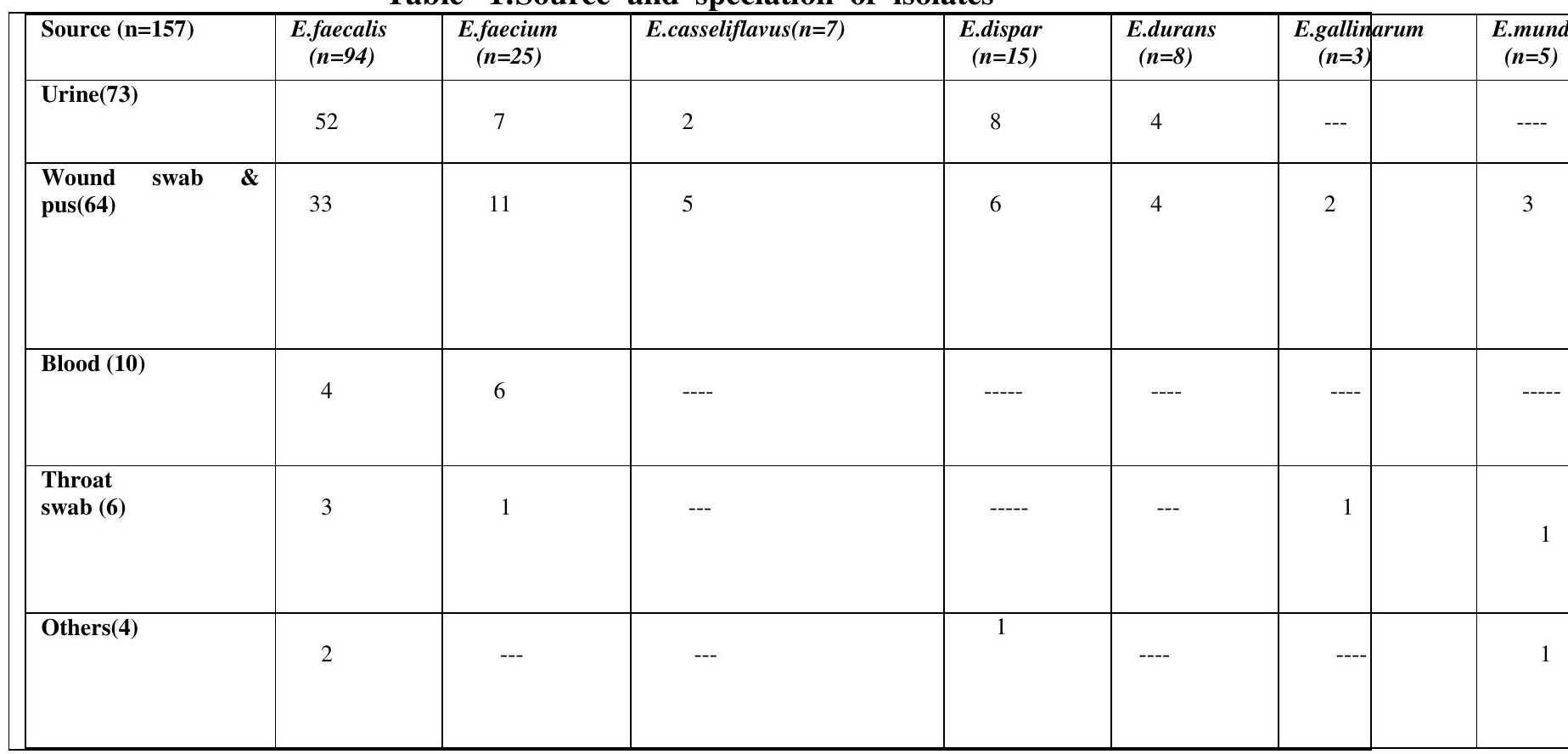

Table--2:Antibiotic resistance (\%) in enterococci by Kirby -Bauer disk diffusion method

\begin{tabular}{|l|c|c|c|l|l|l|}
\hline Antibiotic & $\begin{array}{c}\text { E.faecalis } \\
(\mathbf{N = 9 4})\end{array}$ & $\begin{array}{c}\text { E.faecium } \\
(\mathbf{n = 2 5})\end{array}$ & $\begin{array}{c}\text { E.dispar } \\
(\mathbf{n = 1 5})\end{array}$ & $\begin{array}{c}\text { E.casseliflav. } \\
(\mathbf{n = 7})\end{array}$ & $\begin{array}{c}\text { E.durans } \\
(\mathbf{n = 8})\end{array}$ & $\begin{array}{c}\text { E.gallinarum } \\
(\mathbf{n = 3})\end{array}$ \\
\hline Penicillin & 76.5 & 88 & 40 & 57.1 & 50 & 32.75 \\
\hline Amoxycillin & 69.1 & 80 & 33.3 & 57.1 & 37.5 & 66.6 \\
\hline Amoxyclave & 55.3 & 72 & 20 & 42.8 & 25 & 33.3 \\
\hline Ceftazime & 51.06 & 60 & 13.33 & 28.5 & 37.5 & 33.3 \\
\hline Azithromycin & 46.8 & 56 & 26.6 & 42.8 & 62.5 & 66.6 \\
\hline Piperacilln & 44.8 & 52 & 33.3 & 71.4 & 37.5 & 33.3 \\
\hline Imipenem & 42.7 & 68 & 33.3 & 57.1 & 62.5 & 0 \\
\hline Nitrofurantoin & 40.42 & 48 & 20 & 42.8 & 25 & 33.3 \\
\hline Vancomycin & 28.72 & 40 & 40 & 100 & 25 & 100 \\
\hline Teicoplanin & 2.12 & 0 & 0 & 0 & 0 & 0 \\
\hline Linezolid & 0 & 0 & 0 & 0 & 0 & 0 \\
\hline
\end{tabular}

E.mundtii (05) strains were found to be (100\%)sensitive to all the antibiotics tested. 
Table-3: HLAR InDifferent Species OfEnterococi

\begin{tabular}{|l|l|l|l|l|}
\hline $\begin{array}{l}\text { Enterococcus } \\
\text { Species }\end{array}$ & Total number isolated & $\begin{array}{l}\text { HLGR strains } \\
(\%)\end{array}$ & $\begin{array}{l}\text { HLSR strains } \\
(\%)\end{array}$ & $\begin{array}{l}\text { Combined } \\
\text { Resistance }(\%)\end{array}$ \\
\hline E. faecalis & 94 & $34(36.1 \%)$ & $30(31.9 \%)$ & $28(21.2 \%)$ \\
\hline E. faecium & 25 & $25(100 \%)$ & $22(88 \%)$ & $22(88 \%)$ \\
\hline E.casseliflavus & 07 & $02(28.5)$ & $01(14.2)$ & $01(14.2 \%)$ \\
\hline E.disper & 15 & $\mathrm{~N}$ & $\mathrm{~N}$ & $\mathrm{~N}$ \\
\hline E.durans & 08 & $02(25 \%)$ & $02(25 \%)$ & $02(25 \%)$ \\
\hline E.gallinarum & 03 & $\mathrm{~N}$ & $\mathrm{~N}$ & $\mathrm{~N}$ \\
\hline E. mundtii & 05 & $\mathrm{~N}$ & $\mathrm{~N}$ & $\mathrm{~N}$ \\
\hline Total & 157 & 63 & 55 & 53 \\
\hline
\end{tabular}

$\mathrm{N}$-nil, HLAR---High level aminoglycoside resistance, HLSR---High level Streptomycin resistance, HLGR--High level Gentamicin resistance.

Table--4: Phenotypes of glycopeptides-resistant enterococci

\begin{tabular}{|l|l|l|l|l|l|}
\hline Species & Total number & Phenotype & $\begin{array}{l}\text { Vancomycin } \\
\text { MIC }(\mu \mathrm{g} / \mathrm{ml})\end{array}$ & $\begin{array}{l}\text { Teicoplanin } \\
(\mu \mathrm{g} / \mathrm{ml})\end{array}$ & $\begin{array}{l}\text { M0de of acquisition } \\
\text { of resistance }(\mu \mathrm{g} / \mathrm{ml})\end{array}$ \\
\hline E.faecalis & $\mathbf{0 2}$ & VanA & $\mathbf{2 5 6}$ & $\mathbf{1 6}$ & Acquired \\
\hline E.faecium & $\mathbf{0 9}$ & VanB & $\mathbf{1 6 - 3 2}$ & $<\mathbf{0 . 5}$ & Acquired \\
\hline E.casseliflavus\&E.gallinarum & $\mathbf{0 9}$ & VanC & $\mathbf{0 2 - 0 4}$ & $<0.125$ & Intrinsic \\
\hline
\end{tabular}

\section{Discussion}

There is a growing awareness of the public health concerns associated with the occurrence of drug resistant strains of bacteria. The emergence of multiple antibiotic resistant bacteria has become a major challenge in the treatment of infectious disease[15,16].Enterococci are recognized as important human pathogen in both community and hospital acquired infections[2,15].Recent years have witnessed increased interest not only because of their ability to cause serious infections but also because of their increasing resistance to many antimicrobial agents[2, 17].In the present study various species of enterococci isolated are----E. faecalis 94(59.87\%), E. faecium 25(15.92\%), E. dispar15(9.55\%), Edurans8(5.0\%), E. casseliflavus 7(4.45\%), E mundtii 5(3.18\% ),E.gallinarum3(1.91\%)[Table-1]which corroborates with the findings of Desai PJ et al , Bhat $\mathrm{KG}$ et al and others from India[1,8].In contrast, only two species were recovered by Karmarkar MG et al,Mendiratta DK et al and Ghoshal et al[18, 19]. In our study predominant species isolated areE. faecalis followed by E. faecium which is similiar to the reports of other studies from India[17,20]. Reasons could be the predominance of E. fecalis in the endogenous flora of the body[20,21] .However in some studies E.faecium is found as the most prevalent species incongruence with our studies[18,19]. Our isolation rate(E.faecalis$59.87 \%$ ) is close to that ofAdhikary $\mathrm{L}($ E.faecalis-72.22\%) although a higher rate of isolation of E.faecalis has been reported by Mendiratta DK et al(85.3\%),Agarwal VA et al from Nagpur(86\%) and Parvathi S et al from Coimbatore( $88 \%)$.But the isolation rate of E.faecium(15.92\%) in our study well corroborates with the studies from central India(14.7\%) and Nagpur (14\%).

Species identification of isolates enabled to assess the species specific susceptibility patterns.In this study resistance to different antibiotics are more among E.faecium than E.faecalis which is of clinical importance as it limits the therapeutic options.It is noteworthy thatmajority of uncommon species identified in our study are mainly recovered from community acquired infections which exhibitsincreased susceptibility to various antibiotic except few strains of E.casseliflavus and E.disparwhich showed increased resistance to different antibiotics and were nosocomial in origin.

Among aminoglycosides, $100 \%$ ofthe isolates exhibited resistance to gentamicin $(80 \mu \mathrm{g})$ bydisc diffusion method. In the present study, $41.4 \%$ of the enterococci showed HLAR and HLAR was more among E. faecium than E.faecalis [Table-4] as has been reported previously also[11,12]. Combined HLGR and HLSR was significantly $(\mathrm{p}=0.002, \chi=14.69)$ higher in E.faecium (88\%) than E.faecalis $(21.2 \%)$ which corroborates with the reports of Mendiratta DK et al and Gordon et al. Both HLGR and HLSR was seen in 53isolates.HLAR in these strains can well nullify the efficacy of combination therapy. Therefore distinguishing HLAR from simple intrinsic resistance is important and should be adopted as a part of routine Microbiology laboratory.

In this study, the phenotypic classification of Vancomycin resistant enterococci(VRE) showed two(02) VanA phenotypes with raised MIC values to both Vancomycin(MIC--256 $\mu \mathrm{g} / \mathrm{ml}$ ) and Teicoplanin(MIC-$16 \mu \mathrm{g} / \mathrm{ml}$ ) whereas the other nine(09) were VanB phenotypes with raised MIC to Vancomycin (MIC16-32 $\mu \mathrm{g} / \mathrm{ml}$ ) but susceptible to Teicoplanin (MIC $<0.5 \mu \mathrm{g} / \mathrm{ml}$ ). The uncommonenterococcalstrains $(09)$ were categorized as VanC phenotype which had intrinsic low level resistance to Vancomycin(MIC2-4 $\mu \mathrm{g} / \mathrm{ml}$ ) but sensitive to 
Teicoplanin. Surprisingly one E.casseliflavus strain would have shown VanC phenotype was found to be sensitive to glycopeptides. The reason may be due to technical error in identification.

The results of our study are based on phenotypic methods alone. It was felt earlier that the use of both phenotypic and genotypic in conjunction with each other would provide a more accurate information[10,11]. Nevertheless, this phenotypic classification is useful, because it usually corresponds well with the genotypic classification and utilizes information that can be derived simply and inexpensively in a laboratory[3,22].

The two VRE strains identified as VanA phenotype were isolated from blood and urine sample of patients admitted in paediatricward. Surprisingly from the record it was found that two samples(blood, urine) were obtained from the same patient in two different occasions. Repeat samples also confirmed the same results. The enterococcal strain was identified as E.faecalis which is VanA phenotype with MIC for vancomycin as high as $256 \mu \mathrm{g} / \mathrm{ml}$. Thepatient was a one and half year old male child admitted with FUO(Fever of unknown origin) and occasional pain abdomen for last two weeks. He had a prior history of hospitalization for respiratory distress due to severe RTI one month back. The isolated and identified $\boldsymbol{E}$.faecalis from both the samples showed resistance to all the antibiotics tested except Linezolid and Tigecycline. The strain also showed HLAR to both streptomycin and gentamicin. The patient was managed conservatively and was administered linezolid to which he responded well. The risk factor associated in this case were history of previous hospitalization, prolonged antibiotic treatment.

Previously from India, there are few reports of emergence of vancomycin resistance in enterococcal strains with increased MIC values [Table-5] [23,24,18,25,19,20,26,27]. The VanA strain isolated in our case had MIC values for vancomycin ashigh as $256 \mu \mathrm{g} / \mathrm{ml}$ and teicoplaninas $16 \mu \mathrm{g} / \mathrm{ml}$.

We conclude that enterococcal strains with high rate of resistance to aminoglycoside and other antimicrobial agents are prevalent in our hospital settings and emergence of VRE has again worsened the situation.This signals reconsideration of antibiotic policies by the Infection control committee(ICC) and urgent control measures should be adopted to prevent spread of such infection

Table--5: VRE Isolation : Indian scenario

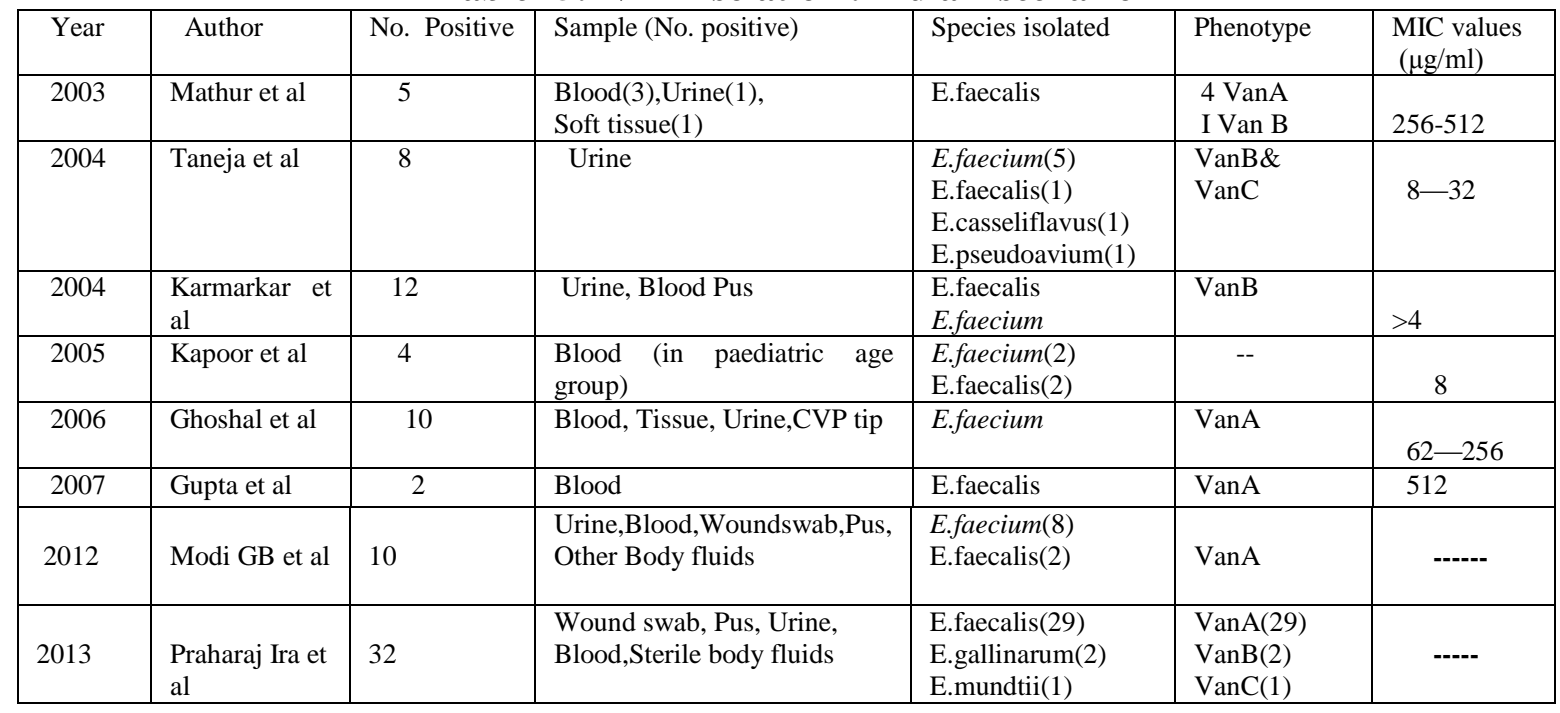

\section{References}

[1]. Desai PJ, Pandit D, Mathur M, Gogate A. Prevalence, identification and distribution of variousspecies of enterococci isolated from clinical specimens with special reference tourinary tract infection in catheterized patients. Indian J Med Microbiol 2001; 19: 1327.

[2]. Murray BE. The life and times of enterococci. ClinMicrobiol Rev 1990;3:46-65.

[3]. SoodSeema, MalhotraMeenakshi, Das BK, KapilArti.Enterococcal infections \& antimicrobial resistance. Indian J Med Res 2008; 128: 111-121.

[4]. Edwards DD, Enterococci attracts attention of concerned microbiologists.ASM News 2000;66: 540-5.

[5]. Cetinkaya Y, Falk P, Mayhall G, Vancomycin resistant enterococci. Clin Microbial Rev 2000;13: 686-707.

[6]. Jones RN, Sader HS, Erwin ME, Anderson SC and Enterococcus Study group. Emerging multiple resistant enterococci. DiagnMicrobiol Infect Dis 1995; 21: 85-9

[7]. Liassine N, Frei R, Jan I, Auckenthaler R. Characterization of gltcopeptide resistant enterococci from a Swiss hospital. $J$ ClinMicrobiol 1998; 36: 1853-58.

[8]. Sham DF, Fre L, Smith C, Eveland M, Mundy LM. Rapid characterization schemes for surveillance of isolates of vancomycin resistant enterococci. J ClinMicrobiol 1997; 35: 2026-30.

[9]. Low DE, Keller N, Barth A, Jones RN. Clinical prevalence, antimicrobial susceptibility and geographic resistance patterns of enterococci: results from the SENTRY AntimicrobialSurveillance Program, 1997-1999. Clin Infect Dis 2001; 32 (Supply 2) :S133-45. 
[10]. Nelson RRS, McGregor KF,Brown AR, Amyes GB, Young HK. Isolation and characterization of glycopeptides resistant enterococci from hospitalized patients over a thirty month period. J ClinMicrobiol2000; 38 : 2112-6.

[11]. Schaberg DR, Culver DH, Gaynes RP. Major trends in the microbial etiology of nosocomial infection. Am J Med 1991; 91 :72S$75 \mathrm{~S}$.

[12]. Facklam RR, Collins MD. Identification of enterococcus species isolated from human infections by a conventional test scheme. $\mathbf{J}$ ClinMicrobiol 1989; 27:731-4.

[13]. Clinical and Laboratory Standards Institute. Performance Standards for Antimicrobial Susceptibility Testing ; Twenty-third Informational supplement. CLSI document M100 S-23 vide M02 A11. 950 West Valley Road,Wayne,Pennsylvania,USA 2013.

[14]. Clinical and Laboratory Standards Institute. Performance Standards for Antimicrobial Susceptibility Testing; Twenty-third Informational supplement.CLSI document M100 S-23 vide M07 A9. 950 West Valley Road, Wayne, Pennsylvania.USA 2013

[15]. Rice EW, Messer jw, Johnson CH, Reasoner DJ. Occurrence of high level aminoglycoside resistance in environmental isolates of enterococci.Applied and Environmental Microbiology 1995; 61(1) :374-76.

[16]. Davies J. Inactivation of antibiotics and the dissemination of resistance genes. Science 1994; 264: $375-82$

[17]. Mendiratta DK, Kaur H, Deotale V, Thamke DC, Narang R, Narang P. Status of high level aminoglycoside resistant Enterococcus faecium and Enterococcus faecalis in a rural hospital of central India. Indian J Med Microbiol 2008; 26 : $369-71$.

[18]. Karmarkar MG, Gersham ES, Mehta PR. Enterococcal infections with special reference to phenotypic characterization and drug resistance.Indian J Med Res 2004; $119: 22-5$.

[19]. Ghoshal U, Garg A, Tiwari DP, Ayyagiri A. Emerging vancomycin resistance in Enterococci in India. Indian J PatholMicrobiol 2006; 49(4) : 620-22.

[20]. Gupta V, Singla N. Antibiotic susceptibility pattern of enterococci. Journal of Clinical and Diagnostic Research [serial online] 2007; 5(4) : 385-89.

[21]. Hall LMC. Recent advances in understanding the epidemiology of Enterococci. Rev Med Microbiol 1993; 4 : 192-97.

[22]. Eliopoulos GM. Vancomycin-resistant enterococci. Mechanism and clinical relevance.Infect Dis Clin North Am 1997;11: 851-65.

[23]. Mathur P, Kapil A, Chandra S, Sharma P, Das B.Antimicrobial resistance pattern in Enterococcus faecals at a tertiary care centre of northern India. IndianJ Med Res 2003;118:25-8.

[24]. Taneja N, Rani P, Emmanual R, Sharma M. Significance of vancomycin resistant Enterococci from urinary specimen at a tertiary care centre in northern India. Indian J Med Res 2004;119:72-4.

[25]. Kapoor L, Randhawa,VS, Deb M. Antimicrobial resistance of Enterococcal blood isolates atapaediatic care hospital in India. Jpn J Infect Dis 2005;58:101-3.

[26]. Modi GB, Soni ST, Patel J, Goswami HM, Vegad MM. Prevalence of vancomycin resistant Enterococci in tertiary care hospital,Western,India.International Journal of Microbiologyy Research 2012; 4(2) : 182-85.

[27]. Praharaj Ira, Sujatha S,ParijaSubhash C. Phenotypic and genotypic characterization of vancomycin resistant Enterococcus isolates from clinical specimens. Indian J Med Res 2013; 138(4): 549-56.

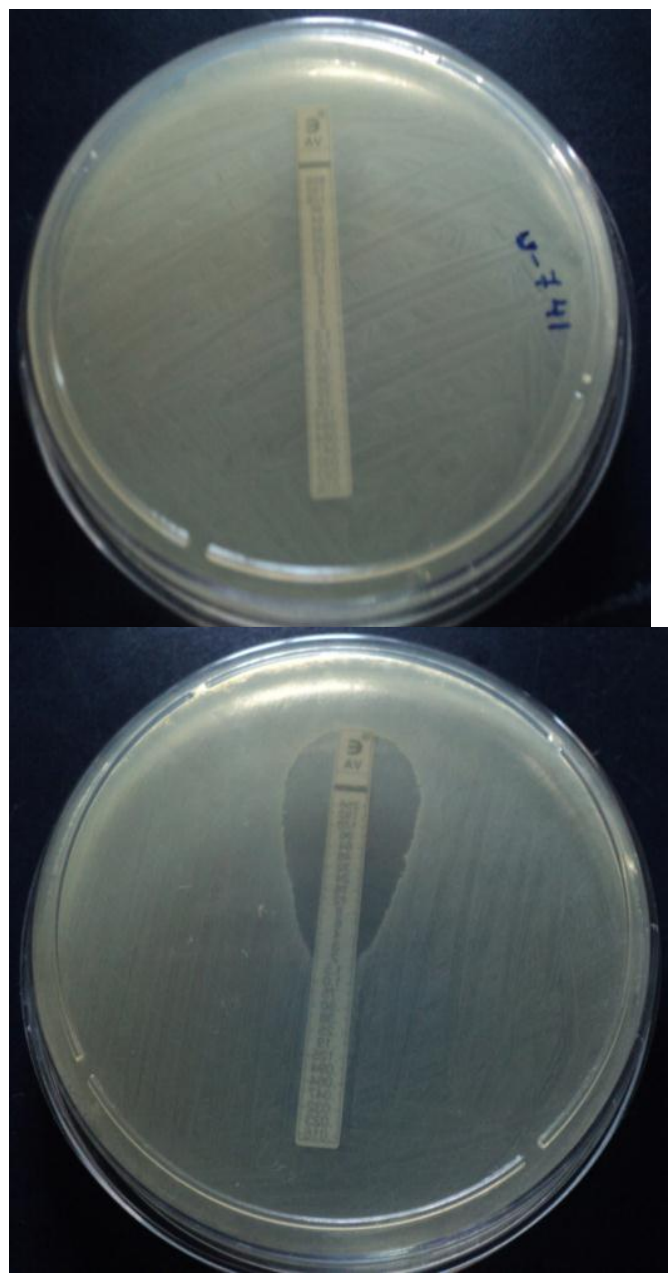

Figure 1: VanA phynotype showing Vancomycine resistance by $\mathrm{E}$ - test. (MIC $256 \mu \mathrm{g} / \mathrm{lit}$ ).

Figure 2: VanC phynotype showing Vancomycine resistance by $\mathrm{E}$ - test. (MIC $>2 \mu \mathrm{g} / \mathrm{lit}$ ) 